\title{
DETERMINAÇÃO DO BORO, SOLUVEL EM ÁGUA, EM FERTILIZANTES *
}

\author{
J. C. Alcarde ** \\ R. A. Catani ** \\ F. M. KROLL ***
}

\begin{abstract}
O presente trabalho relata os estudos desenvolvidos sôbre o método volumétrico de determinação do boro, solúvel em água, em fertilizantes, que se fundamenta na titulação do ácido bórico ou borato por uma solução padronizada de $\mathrm{NaOH}$, em presença de manitol.

Os estudos objetivaram simplificar algumas das técnicas preconizadas, estabelecer a quantidade de manitol efetivamente necessária e examinar a influência da uréia. Os resultados mostraram que apenas 6,0 $\mathrm{g}$ de manitol são necessários para a determinação, quantidade essa suficiente para titular 2,5 equivalentes miligramas ou milimoles de $\mathrm{H}_{3} \mathrm{BO}_{3}$. Também o método não é influenciado pela uréia, podendo ser aplicado em fertilizantes contendo até $20 \%$ dêsse composto nitrogenado.
\end{abstract}

\section{INTRODUÇÃO}

Os micronutrientes que, de uma maneira mais generalizada, se mostram carentes em solos do Estado de São Paulo e regiões próximas são o zinco e o boro. Tem sido assinalada deficiência de boro em várias culturas e em diversas regiões (BRASIL SOBR. ${ }^{\circ}, 1965$; MALAVOI TA \& OUTROS, 1967).

Uma das técnicas de correção de deficiências de micronutrientes é o uso de misturas de fertilizantes que os contém. Tal técnica apresenta como vantagens' o fato da distribuição ser mais uniforme e de diminuir o número de aplicações relativas à adubação (NELSON \& TERMAN, 1963). O boro, juntamente com o zinco, é o micronutriente mais comumente adicionada às misturas de fertilizantes.

Para a determinação do boro em fertilizantes diversos métodos têm sido propostos: métodos colorimétricos (BORLAND, BROWNLIE \& GODDEN, 1967), métodos baseados na fotometria de chama de absorção atômica (MELTON, HOOVER \& HOWARD, 1969; WE-

\footnotetext{
* Entregue para a publicação em 28-12-1971.

** Departamento de Química da E.S.A. "Luiz de Queiroz".

*** Bolsista do Conselho Nacional de Pesquisas.
} 
GER, HOSSNER \& FERRARA, 1970 ; GEHRKE, 1971)e métodos baseados na fotometria de chama de emissão (PICKETT, PAU \& KOIRTYOHANN, 1971). Atualmente, êsses métodos físico-químicos estão sendo propostos para serem objeto de estudos mais aprimorados (SCHALL, 1969; RUND, 1970 e 1971). No entanto o método mais empregado ainda é o volumétrico, baseado na clássica titulação do ácido bórico ou borato com uma solução padronizada de $\mathrm{NaOH}$, em presença de um poliálcool (manitol ou sorbitol). Esse método é considerado oficial nos Estados Unidos (OFFICIAL METHODS OF ANALYSIS OF T'HE A.O.A.C. 1965) e já foi estudado por diversos autores (TAYLOR, 1949; BERRY, 1953 e 1955; BORLAND; BROWNLIE \& GODDEN, 1967).

O presente trabalho relata os estudos desenvolvidos sôbre o referido método com a finalidade de simplificar algumas das técnicas recomendadas, estabelecer a quantidade de manitol efetivamente necessária e estudar a influência da uréia na citada determinação.

\section{MATERIAL E MÉTODOS}

\section{Material}

O material constou de duas misturas de fertilizantes, caracterizadas como $\mathrm{M}_{1}$ e $\mathrm{M}_{2}$, preparadas em laboratório a partir de fertilizantes simples comerciais. O preparo das misturas foi feito colocando-se todos os componentes num gral de porcelana, onde se procedeu a trituração e homogeneização. $O$ quadro 1 apresenta a composição porcentual de cada mistura.

QUADRO 1 - Composição porcentual das misturas de fertilizantes, preparadas em laboratório.

\begin{tabular}{lcc}
\hline Componentes & $\mathbf{M}_{\mathbf{1}}$ Misturas & $\mathbf{M}_{2}$ \\
\hline Superfosfato simples & 20 & 30 \\
Superfosfato triplo & 30 & 30 \\
Cloreto de potássio & 20 & 20 \\
Salitre do Chile & 10 & 10 \\
Sulfato de amônio & 20 & 10 \\
\hline
\end{tabular}

A fórmula $\left(\% \mathrm{~N}-\% \mathrm{P}_{2} \mathrm{O}_{5}-\% \mathrm{~K}_{2} \mathrm{O}\right)$ aproximada da mistura $\mathrm{M}_{1}$ é 5,5 - 17,0 - 12,0 e da mistura $M_{2}$ é 3,5 - 20,0-12,0.

De cada mistura foram preparadas amostras contendo em tôrno de $1,5 \%, 3,0 \%$ e $6,0 \%$ de $\mathrm{H}_{3} \mathrm{BO}_{3}$ p.a., que corresponde aproximadamente a $0,25 \%, 0.5 \%$ e $1,0 \%$ de boro. A porcentagem mínima de $0,25 \%$ de boro foi adotada em face do que menciona NELSON \& TERMAN (1963). 
Não constituiu preocupação a obtenção de uma concentração exata de $\mathrm{H}_{3} \mathrm{BO}_{3}$ adicionado porquanto ela se altera em virtude da higroscopicidade das misturas e, segundo TAYLOR (1949), há a possibilidade da formação de boratos de cálcio e borofosfatos pouco solúveis, durante o armazenamento.

\section{Reativos}

Dentre os reativos utilizados, merecem menção os seguintes:

Solução padrão de $\mathbf{H}_{3} \mathbf{B O}_{3} \mathbf{0 , 1 0 0} \mathbf{N}$. - Dissolver $3,0918 \mathrm{~g}$ de $\mathrm{H}_{3} \mathrm{BO}_{3}$ p.a. sêco em estufa a $40^{\circ} \mathrm{C}$ por 4 horas, em água destilada, transferir para balão volumétrico de $500 \mathrm{ml}$ e completar o volume com água destilada.

Solução de $\mathrm{NAOH}$, aproximadamente $0,05 \mathrm{~N}$, livre de $\mathrm{CO}_{2}-$ Ferver 3 litros de água por 20 minutos, com a finalidade de remover $\mathrm{CO}_{2}$, esfriar ràpidamente e transferir para um frasco de plástico fechado com dispositivo contendo "ascarite" para evitar a entrada do $\mathrm{CO}_{2}$ do ar. Juntar $15 \mathrm{ml}$ do sobrenadante de uma solução $10 \mathrm{~N}$ de $\mathrm{NaOH}$ (livre de $\mathrm{Na}_{2} \mathrm{CO}_{3}$ ) e agitar. Ligar o frasco de plástico a uma bureta, na qual também deve ser adaptado um dispositivo contendo "ascarite" para evitar a entrada do $\mathrm{CO}_{2}$ do ar. Padronizar a referida solução.

\section{Métodos}

\section{Padronização da solução aproximadamente 0,05 $\mathrm{N}$ de $\mathrm{NaOH}$}

a) Transferir 3 vêzes $0,2500 \mathrm{~g}$ de ftalato ácido de potássio (padrão primário), para frascos de Erlenmeyer de $250 \mathrm{ml}$. Juntar $50 \mathrm{ml}$ de água destilada, agitar até dissolver o sal e acrescentar 2 a 3 gôtas de solução alcoólica de fenolftaleina a $1 \%$.

b) Encher a bureta, especialmente instalada, com solução aproximadamente $0,05 \mathrm{~N}$ de $\mathrm{NaOH}$, livre de $\mathrm{CO}_{2}$.

c) Titular a solução do frasco de Erlenmeyer até o aparecimento de uma côr levemente rosada.

\section{Estudo sôbre a quantidade de manitol}

MELLON \& MORRIS (1924) estudaram a eficiência de diversos poliálcoois e açúcares na complexação do ácido bórico, concluindo que o manitol é o mais eficiente dos poliálcoois e o açúcar invertido o mais eficiente dos açúcares.

A quantidade de manitol recomendada para a titulação do ácido bórico com solução de $\mathrm{NaOH}$ tem sido bastante variável. MELLON \& MORRIS (1924) estabeleceram a curva de neutralização de uma solução $0,1 \mathrm{~N}$ de $\mathrm{H}_{3} \mathrm{BO}_{3}$ por uma solução $0,2 \mathrm{~N}$ de $\mathrm{NaOH}$, empregando 4 moles do poliálcool por mól de $\mathrm{H}_{3} \mathrm{BO}_{3}$. SCOTT (1939) recomenda 4 
e $8 \mathrm{~g}$ de manitol, para a determinação do boro em boratos solúveis em água, empregando soluções $0,5 \mathrm{~N}$ e $1,0 \mathrm{~N} \cdot$ de $\mathrm{NaOH}$, respectivamente. KOLTHOFF \& STENGER (1947) relatam que são necessários 0,5 g a $0,7 \mathrm{~g}$ de manitol para titular $10 \mathrm{ml}$ de solução $0,1 \mathrm{~N}$ de $\mathrm{H}_{3} \mathrm{BO}_{3}$.

No método volumétrico de determinação do boro em fertilizantes, os quais apresentam teores relativamente baixos dêsse elemento, a quantidade recomendada de manitol é de $20 \mathrm{~g}$ (OFFICIAL METHODS OF ANALYSIS OF THE A.O.A.C., 1965), quantidade essa já empregada por BERRY (1953 e 1955).

Em face disso, procurou-se estabelecer a quantidade efetivamente necessária do referido poliálcool na presente determinação, considerancio o equilíbrio que se estabelece na formação do complexo ácido bórico-manitol (DEUTSCH \& OSOLING, 1949; ROSS \& CATTOTI, 1949; NIELS \& CAMPBELL, 1964 ; NICHERSON, 1968 e 1970 ; CAMPBEILL Jr., 1969; BELCHER, 1970). O estudo foi desenvolvido usando quantidades variáveis de manitol para titular $25 \mathrm{ml}$ de solução 0,100 $\mathrm{N}^{\prime}$ de $\mathrm{H}_{3} \mathrm{BO}_{3}$.

\section{Procedimento}

a) Transferir $25 \mathrm{ml}$ de solução padrão $0,100 \mathrm{~N}$ de $\mathrm{H}_{3} \mathrm{BO}_{3}$ para frasco de Erlenmeyer de $300 \mathrm{ml}$ e juntar aproximadamente $150 \mathrm{ml}$ de água destilada.

b) Acrescentar 5-6 gôtas de solução de vermelho de metila a $0,5 \%$ e tornar a solução rósea pela adição de solução de HC1 ( 1 + $5)$.

c) Adicionar 3-4 "boileezers", cobrir com funil e ferver por 5 minutos para remover o $\mathrm{CO}_{2}$. Esfriar em água enquanto coberto e lavar o funil e as paredes do frasco com pequena porção de água destilada.

d) Adicionar, cuidadosamente, solução de $\mathrm{NaOH} 0,05 \mathrm{~N}$, livre de $\mathrm{CO}_{2}$, até obter-se a côr amarela do vermelho de metila.

e) Juntar manitol. No estudo presente foram usados $2 \mathrm{~g}, 4 \mathrm{~g}$, $6 \mathrm{~g}$ e $20 \mathrm{~g}$ de manitol. Agitar até a sua completa dissolução e acrescentar $1 \mathrm{ml}$ de solução de fenolftaleina a $1 \%$.

f) Titular com a solução padronizada de $\mathrm{NaOH}$, livre de $\mathrm{CO}_{2}$, até a obtenção da côr rosada da fenolftaleina. Anotar o volume gasto. Conduzir uma prova em branco.

\section{Determinação do boro, solúvel em água, em fertilizantes}

a) Pesar 2,500 g da amostra finamente moída, transferir para copo de $250 \mathrm{ml}$ e adicionar $125 \mathrm{ml}$ de água destilada.

b) Ferver vagarosamente por 10 minutos e filtrar quente através de papel de filtro S \& S 589, faixa branca, para copos de $400 \mathrm{ml}$. 
c) Lavar o copo e o resíduo com 6 porções de $10 \mathrm{ml}$ de água destilada quente e fazer um volume de aproximadamente $200 \mathrm{ml}$.

d) Aquecer o filtrado até próximo da ebulição e acrescentar $15 \mathrm{ml}$ de solução de $\mathrm{BaCl}_{2}$ a $10 \%$ para precipitar fosfatos e sulfatos. Acrescentar 4 gôtas de solução de fenolftaleina a $1 \%$ e, vagarosamente, adicionar $\mathrm{Ba}(\mathrm{OH})_{2}$, pulverizado ou de uma solução saturada, até a suspensão adquirir côr fortemente rosada.

e) Ferver, com o copo aberto, por 60 minutos, para eliminar $\mathrm{NH}_{3}$. Caso ocorra o descoramento do indicador, acrescentar mais $\mathrm{Ba}(\mathrm{OH})_{2}$. E no final, se necessário, adicionar água para fazer um volume de $200 \mathrm{ml}$.

f) Filtrar através de papel de filtro $S \&$ S 589, faixa branca, para frascos ide Erlenmeyer de $500 \mathrm{ml}$, lavando o copo e o precipitado com 6 porções de $10 \mathrm{ml}$ de água destilada quente e fervida.

g) Tornar o filtradó incolor pela adição de algumas gôtas de HC1 $(1+5)$. Acrescentar 6 gôtas de solução de vermelho de metila a $0,5 \%$ e continuar a adição da solução de ácido até a obtenção da côr rósea do vermelho de metila.

h) Adicionar 3 a 4 "boileezers", cobrir com funil e ferver por 5 minutos para remover $\mathrm{CO}_{2}$.

i) Esfriar enquanto coberto e lavar o funil e as paredes do frasco com pequena quantidade de água destilada.

j) Adicionar, cuidadosamente, solução $0,05 \mathrm{~N}$ de $\mathrm{NaOH}$, livre de $\mathrm{CO}_{2}$, até o aparecimento da côr amanela do vermelho de metila.

k) Adicionar $6,0 \mathrm{~g}$ de manitol, agitar até a completa dissolução e acrescentar $1 \mathrm{ml}$ de solução alcoólica de fenolftaleina a $1 \%$.,

1) Titular com a solução padronizada de $\mathrm{NaOH}$, livre de $\mathrm{CO}_{2}$, até o ponto final róseo. Anotar o volume gasto. Conduzir uma prova em branco.

\section{RESULTADOS OBTIDOS E DISCUSSÃO}

\section{Estudo sôbre a quantidade de manitol}

Os resultados dos estudos desenvolvidos com o objetivo de estabelecer a quantidade de manitol efetivamente necessária à determinação acham-se descritos no quadro 2 . 
QUADRO 2 - Resultados da titulação de $25 \mathrm{ml}$ de solução $0,100 \mathrm{~N}$ de $\mathrm{H}_{3} \mathrm{BO}_{3}$ em função da quantidade de manitol. Média de 3 determinações.

\begin{tabular}{ccc}
\hline $\begin{array}{c}\text { gramas de manitol } \\
\text { usados }\end{array}$ & $\begin{array}{c}\text { mg de } \mathrm{H}_{3} \mathrm{BO}_{3} \\
\text { existentes }\end{array}$ & $\begin{array}{c}\text { mg de } \mathrm{H}_{3} \mathrm{BO}_{3} \\
\text { determinados }\end{array}$ \\
\hline 2,0 & 154,6 & 145,3 \\
4,0 & 154,6 & 153,4 \\
6,0 & 154,6 & 154,6 \\
20,0 & 154,6 & 154,6 \\
\hline
\end{tabular}

Esses resultados indicam que para titular 2,5 equivalentes miligramas ou milimoles de $\mathrm{H}_{3} \mathrm{BO}_{3}$, em solução pura dêsse ácido, são suficientes $6,0 \mathrm{~g}$ de manitol.

Em seguida determinou-sie o conteúdo de boro das amostras de fertilizantes empregando 20,0 g e 6,0 g de manitol. Os resultados estão relatados no quadro 3 .

Conforme se observa, a exemplo do que ocorreu com soluções puras $\mathrm{H}_{3} \mathrm{BO}_{3}$, há perfeita concordância entre os resultados obtidos com 20,0 g e com 6,0 g de manitol. Conclue-se, portanto, que 6,0 g de manitol são suficienties para a presente determinação. 


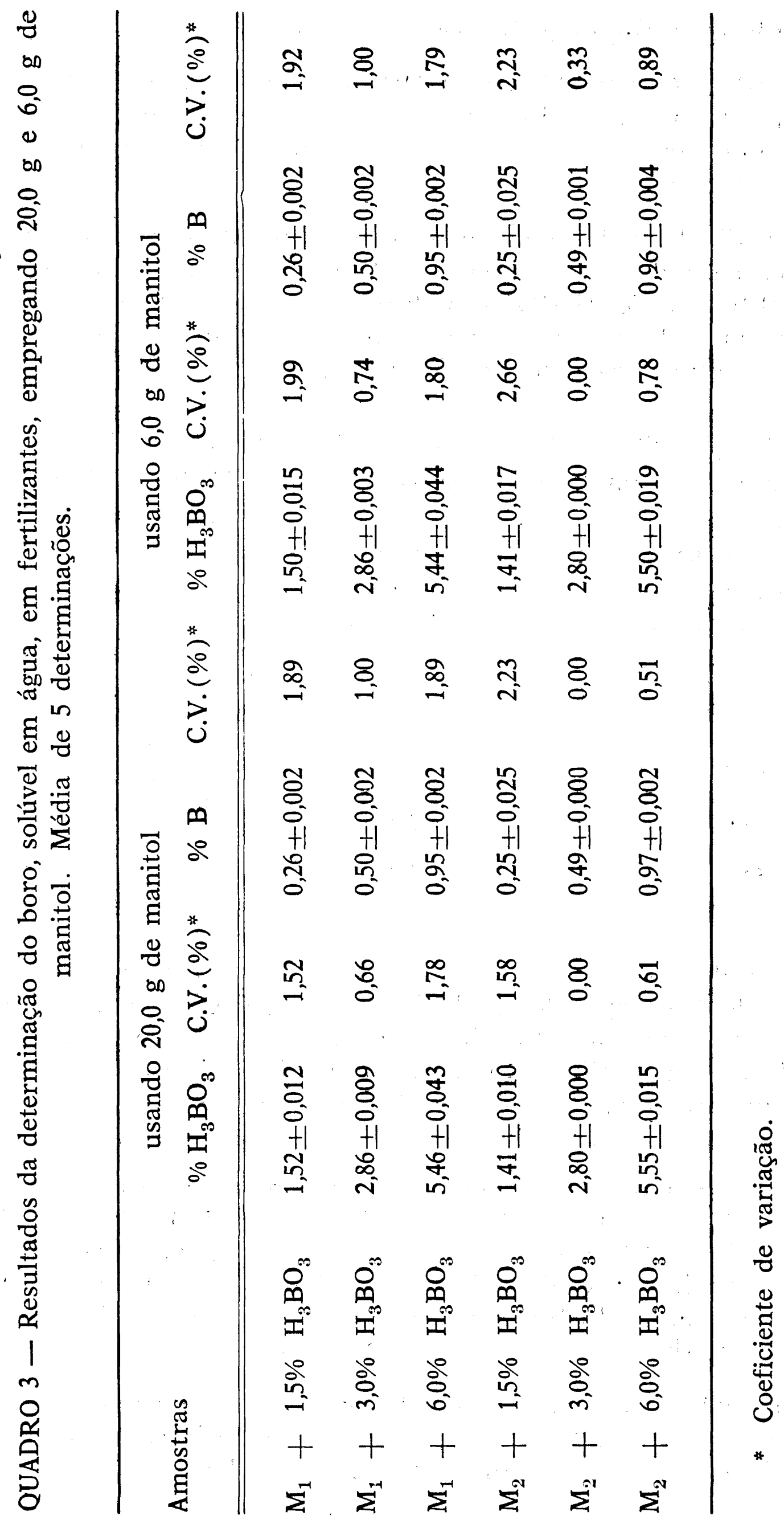




\section{Estudo da influência da uréia}

Segundo o OFFICIAL METHODS OF ANALYSIS OF THE A.O.A.C. (1965), a determinação do boro, solúvel em água, em fertilizantes, pelo método em aprêço, só deve ser feita em amostras contendo, no máximo, $5 \%$ de uréia.

O estudo da influência da uréia foi inicialmente feito usando soluções padrões de ácido bórico e de uréia. Foram usados $25 \mathrm{ml}$ de solução $0,100 \mathrm{~N}$ de $\mathrm{H}_{3} \mathrm{BO}_{3}$ e volumes variáveis de uma solução de uréia contendo $25 \mathrm{mg} / \mathrm{ml}$ e procedeu-se conforme a determinação. Os resultados estão descritos no quadro 4.

QUADRO 4 - Resultados da titulação de $25 \mathrm{ml}$ de solução $0,100 \mathrm{~N}$ de $\mathrm{H}_{3} \mathrm{BO}_{3}$, em presença de quantidades variáveis de uréia. Média de 3 determinações.

\begin{tabular}{ccc}
\hline $\begin{array}{c}\text { mg de } \mathrm{H}_{3} \mathrm{BO}_{3} \\
\text { existentes }\end{array}$ & $\begin{array}{c}\mathrm{mg} \mathrm{de} \mathrm{H}_{3} \mathrm{BO}_{3} \\
\text { colocados }\end{array}$ & $\begin{array}{c}\text { mg de uréia } \\
\text { determinados }\end{array}$ \\
\hline 154,6 & 0 & 154,7 \\
154,6 & 50 & 155,1 \\
154,6 & 125 & 155,4 \\
154,6 & 250 & 155,3 \\
154,6 & 500 & 154,3 \\
\hline
\end{tabular}

Os resultados permitem concluir que, em solùção pura, a determinação alcalimétrica do ácido bórico, em presença de manitol, não é afetada por uma quantidade de uréia de até $500 \mathrm{mg}$. Na determinação do boro em fertilizantes, essa quantidade de uréia corresponde a contida na amostra de 2,500 g de uma mistura apresentando $20 \%$ de uréia.

Posteriormente, estudou-se a influência da uréia usando fertilizantes. Das misturas $\mathrm{M}_{2}+1,5 \%$ de $\mathrm{H}_{3} \mathrm{BO}_{3}, \mathrm{M}_{1}+3,0 \%$ de $\mathrm{H}_{3} \mathrm{BO}_{3}$ e $\mathrm{M}_{1}+6,0 \%$ de $\mathrm{H}_{3} \mathrm{BO}_{3}$, foram preparadas amostras contendo, aproximadamente, $0 \%, 2 \%, 5 \%, 10 \%$ e $20 \%$ de uréia. Procedeuse a determinação do boro nessas misturas e os resultados são mostrados no quadro 5.

De acôrdo com êsses resultados pode-se concluir que a uréia não influi no presente método de determinação do boro, solúvel em água, em fertilizantes, mesmo que êstes a contenha numa quantidade de até $20 \%$. 
QUADRO 5 - Resultados da determinação do boro solúvel em água, expressos em porcentagem de $\mathrm{H}_{3} \mathrm{BO}_{3}$, em fertilizantes contendo diferentes teores de uréia.

\begin{tabular}{lllllll}
\hline \multirow{2}{*}{ Misturas } & \multicolumn{5}{c}{ Porcentagem de uréia nas misturas } \\
\cline { 2 - 7 } & 0 & 2 & 5 & 10 & 20 \\
\hline $\mathrm{M}_{2}+1,5 \%$ & $\mathrm{H}_{3} \mathrm{BO}_{3}$ & 1,42 & 1,39 & 1,39 & 1,39 & 1,43 \\
$\mathrm{M}_{1}+3,0 \%$ & $\mathrm{H}_{3} \mathrm{BO}_{3}$ & 2,77 & 2,77 & 2,84 & 2,77 & 2,79 \\
$\mathbf{M}_{1}+6,0 \% \mathrm{H}_{3} \mathrm{BO}_{3}$ & 5,51 & 5,51 & 5,59 & 5,56 & 5,59 \\
\hline
\end{tabular}

\section{CONCLUSÕES}

Os resultados obtidos permitiram as seguintes conclusões :

a) O método volumétrico de determinação do boro, solúvel em água, em fertilizantes é simples ie sensível, mas relativamente moroso.

b) Apenas 6,0 g de manitol são necessários para a determinação pelo método apresentado. Essa quantidade de manitol é suficiente para titular 2.5 equivalentes miligramas ou milimales de $\mathrm{H}_{3} \mathrm{BO}_{3}$.

c) O método não é influenciado pela uréia, podendo ser aplicado em fertilizantes contendo até $20 \%$ dêsse composto nitrogenado.

\section{SUMMARY}

\section{DETERMINATION OF SOLUBLE BORON IN FERTILIZERS}

This work describes the studies on the volumetric method of water soluble boron determination in fertilizars, by titration of boric acid or borate with standardized $\mathrm{NaOH}$ solution, in presence of mannitol.

The results allowed to conclude that it os necessary only $6.0 \mathrm{~g}$ of mannitol for the determination. This mannitol quantity is enough to titrate 2.5 miliequivalent or milimoles of $\mathrm{H}_{3} \mathrm{BO}_{3}$. Also, this present method is not influenced by urea and this application could by made in fertilizers containing up to $20 \%$ of urea.

\section{LITERATURA CITADA}

BELCHER, R., 1970. A comparative study of various complexing agents (polyols) used in the titration of boric acid. Anal. Chim. Acta, 50(2):261-267.

BERRY, R. C. 1954. Report on boron in mixed fertilizers. J. Ass. Off. Agr. Chem., $36(3): 623-628$.

BERRY, R. C. 1953. Report on boron in mixed fertilizers. J. Ass. Off. Agr. Chem., $37(2): 407-413$.

BORLAND, H., I. A. BROWNLIE \& P. T. GODDEN, 1967. The determination of boron in fertilizers. Analyst, 92:47-53. 
BRASIL SOBR..$^{\circ}$ M. O. C., 1965. Levantamento do teor de boro em alguns solos do Estado de São Paulo. Tese apresentada à E.S.A. "Luiz de Queiroz" para obtenção do título de Docente-Livre USP. Piracicaba 135 pp. mimeografadas.

CAMPBELL, Jr. G. W., 1969. Combining ratio of boric acid and alkali borate with mannitol. J. Inorg. Nucl. Chem., 31(8):2625-2627.

DEUTSCH, A. \& S. OSOLING, 1949. Conductometric and potenciometric studies of the stoichiometric and equilibria of boric mannitol complexes. J. Amer. Chem. Soc., 71(2):1637-1640.

GEHRKE, C. W., 1971. Fertilizers. Anal. Chem. (Annual Rewiew) 43(5):64 R $69 \mathrm{R}$.

KOLTHOFF, I. M. \& V. A. STENGER, 1947. Volumetric Analysis. Vol. II, 2nd ed. Interscience Publishers Inc., New York. 374 pp. Inc. New York. 374 pp.

MALAVOLTA, E., H. P. HAAG, F. A. F. de MELLO \& M. O. C. BRASIL SOBR. ${ }^{\circ}$, 1967. Nutrição Mineral de Algumas Culturas Tropicais. Livraria Pioneira Editôra. Editôra da U.S.P. 251 pp.

MELLON, M. G. \& V. M. MORRIS, 1924. An electrometric study of the titration of boric acid. Ind. and Eng. Chem., 16:123-126.

MELTON, J. R., W. L. HOOVER \& P. A. HOWARD, 1969 - Atomic absorption spectrophotometric determination of water soluble boron in fertilizers. J. Ass. Off. Anal. Chem., 52(2):950-953.

NELSON, W. L. \& G. L. TERMAN, 1963 - Nature, Belavior, and Use of Multinutrient (Mixed) Fertilizers. Em: Fertilizers, Technology and Usage, $p$. 379-427. Edited by M. H. McVickar, G. L. Bridger \& L. B. Nelson. Soil Science Society of America. Wisconsin.

NICKERSON, R. F., 1968 - The combining ratio of boric acid and alkali borate with mannitol. J. Inorg Nucl. Chem., 30:1447-1455.

NIES, N. P. \& G. W. CAMPBELL Jr., 1964 - Inorganic Boron Oxygen Chemistry. Em: Boron Metallo-Boron Compounds and Boranes, p. 53-231. Ed. by R. M. Adams. Interscience Publishers. John Wiley \& Son. New York.

OFFICIAL METHODS OF ANALYSIS OF THE A.O.A.C., 1965. 10th ed., p. 25. Published by the Ass. of Off. Agr. Chem., Washington, D.C.

PICKETT, E. E., J. C. M. PAU \& S. R. KOIRTYOHANN, 1971 - Determination of boron in fertilizers by emission flame photometry in the air-hydrogen flame. J. Ass. Off. Anal. Chem., 54(4):766-800.

ROSS, S. D. \& A. J. CATOTTI, 1949 - Equilibria of the boric acid mannitol complexes. J. Am. Chem. Soc. 71(3):3563-3564.

RUND, R. C., 1970 - Report on fertilizers. J. Ass. Off. Anal. Chem., 53(2):303-304.

RUND, R. C., 1971 - Report on fertilizers. J. Ass. Off. Anal. Chem., 54(2):270-271.

SCHALL, E. D., 1969 - Report on fertilizers. J. Ass. Off. Anal. Chem., 52(2):217218 e $322-323$.

SCOTT, W. W., 1939 - Scott's Stantard Methods of Chemical Analysis. 5th ed., vol. I. Edited by N. H. Furman. D. Van Nostrand Co., Inc., New York. $1234 \mathrm{pp}$.

TAYLOR, D. S., 1949 - Determination of borax in mixed fertilizers. J. Ass. Off. Agr. Chem., 32(2):422-430.

WEGER Jr., S. T., L. R. HOSSNER \& L. W. FERRARA, 1970 - Determination of boron in fertilizers by atomic absorption spectrophotometry. Atomic Absorp. Newsletter, 9(3):58-60. 